\title{
Safety and efficacy of treating symptomatic, partial- thickness rotator cuff tear with fresh, uncultured, unmodified, autologous adipose derived regenerative cells (UA-ADRCs) isolated at point of care: a prospective, randomized, controlled first-in-human pilot study
}

\author{
Jason L. Hurd ${ }^{*}$, Tiffany R. Facile ${ }^{2}$, Jennifer Weiss ${ }^{2}$, Matthew Hayes ${ }^{3}$, \\ Meredith Hayes ${ }^{3}$, John P. Furia ${ }^{4}$, Nicola Maffulli ${ }^{5,6,7}$, Glenn E. Winnier ${ }^{8}$, \\ Christopher Alt ${ }^{9}$, Christoph Schmitz ${ }^{10}$, Eckhard U. Alt ${ }^{2,8,11}$, Mark A. Lundeen, \\ $M D^{12}$ \\ 1 Sanford Orthopedics \& Sports Medicine Sioux Falls, Sioux Falls, SD, USA \\ 2 Sanford Health, Sioux Falls, SD, USA \\ 3 Sanford Radiology Clinic, Sioux Falls, SD, USA \\ ${ }^{4}$ SUN Orthopedics of Evangelical Community Hospital, Lewisburg, PA, USA \\ 5 Department of Musculoskeletal Disorders, Faculty of Medicine and Surgery, University of \\ Salerno, Salerno, Italy \\ ${ }^{6}$ Centre for Sports and Exercise Medicine, Barts and The London School of Medicine and \\ Dentistry, Mile End Hospital, Queen Mary University of London, London, England \\ 7 School of Pharmacy and Bioengineering, Guy Hilton Research Centre, Keele University School \\ of Medicine, Stoke on Trent, England \\ ${ }^{8}$ InGeneron, Inc., Houston, TX, USA \\ 9 InGeneron GmbH, Munich, Germany \\ ${ }^{10}$ Chair of Neuroanatomy, Institute of Anatomy, Faculty of Medicine, LMU Munich, Munich, \\ Germany \\ ${ }^{11}$ Isar Klinikum, Munich, Germany \\ ${ }^{12}$ Sanford Orthopedics \& Sports Medicine Fargo, Fargo, ND, USA
}

\section{Correspondence Jason L. Hurd MD Sanford Orthopedics \& Sports Medicine Sioux Falls 1210 W. 18th St. Suite G01 Sioux Falls South Dakota 57104 \\ USA \\ Phone: +1 (504) 9883040 Mobil: +1 (832) 8533898 E-mail:} Jason.Hurd@SanfordHealth.org
Background: This study tested the hypothesis that treatment of symptomatic, partial-thickness rotator cuff tear (sPTRCT) with fresh, uncultured, unmodified, autologous adipose derived regenerative cells (UA-ADRCs) isolated from lipoaspirate at point of care is safe and more effective than corticosteroid injection. Methods: Subjects aged between 30 and 75 years with sPTRCT who did not respond to traditional nonoperative care for at least six weeks were randomly assigned to receive a single injection of an average $11.4 \times 10^{6} \mathrm{UA}-\mathrm{ADRCs}$ (in $5 \mathrm{ml}$ liquid; mean cell viability: $88 \%)(\mathrm{n}=11$; modified intention-to-treat (mITT) population) or a single injection of $80 \mathrm{mg}$ of methylprednisolone $(40 \mathrm{mg} / \mathrm{ml} ; 2$ $\mathrm{ml})$ plus $3 \mathrm{ml}$ of $0.25 \%$ bupivacaine ( $\mathrm{n}=5$; mITT population), respectively. Safety and efficacy were assessed using the American Shoulder and Elbow Surgeons Standardized Shoulder Assessment Form (ASES), RAND Short Form-36 Health Survey and pain visual analogue scale (VAS) at baseline (BL) as well as 3 weeks (W3), W6, W9, W12, W24, W32, W40 and W52 post treatment. Fat-saturated T2 weighted magnetic resonance imaging of the shoulder was performed at BL as well as at W24 and W52 post treatment.

Results: No severe adverse events related to the injection of UA-ADRCs were observed in the twelve months post treatment. The risks connected with treatment of SPTRCT with UA-ADRCs were not greater than those connected with treatment of sPTRCT with corticosteroid injection. Subjects in the UA-ADRCs group showed statistically significantly higher mean ASES total scores at W24 and W52 post treatment than subjects in the corticosteroid group $(\mathrm{p}<0.05)$.

Discussion: Injection of fresh, uncultured, unmodified, autologous adipose derived regenerative cells is a safe and effective treatment of sPTRCT. Larger trials are needed to verify this result.

Trial registration: Clinicaltrials.gov ID NCT02918136. Registered September 28, 2016.

Level of evidence: Level I; prospective, randomized, controlled trial. 


\begin{abstract}
Background: This study tested the hypothesis that treatment of symptomatic, partial-thickness rotator cuff tear (sPTRCT) with fresh, uncultured, unmodified, autologous adipose derived regenerative cells (UA-ADRCs) isolated from lipoaspirate at point of care is safe and more effective than corticosteroid injection.

Methods: Subjects aged between 30 and 75 years with SPTRCT who did not respond to traditional nonoperative care for at least six weeks were randomly assigned to receive a single injection of an average $11.4 \times 10^{6}$ UA-ADRCs (in $5 \mathrm{ml}$ liquid; mean cell viability: $88 \%)(\mathrm{n}=11$; modified intention-to-treat (mITT) population) or a single injection of $80 \mathrm{mg}$ of methylprednisolone (40 $\mathrm{mg} / \mathrm{ml} ; 2 \mathrm{ml}$ ) plus $3 \mathrm{ml}$ of $0.25 \%$ bupivacaine ( $\mathrm{n}=5$; mITT population), respectively. Safety and efficacy were assessed using the American Shoulder and Elbow Surgeons Standardized Shoulder Assessment Form (ASES), RAND Short Form-36 Health Survey and pain visual analogue scale (VAS) at baseline (BL) as well as 3 weeks (W3), W6, W9, W12, W24, W32, W40 and W52 post treatment. Fat-saturated T2 weighted magnetic resonance imaging of the shoulder was performed at BL as well as at W24 and W52 post treatment.

Results: No severe adverse events related to the injection of UA-ADRCs were observed in the twelve months post treatment. The risks connected with treatment of sPTRCT with UA-ADRCs were not greater than those connected with treatment of sPTRCT with corticosteroid injection. Subjects in the UA-ADRCs group showed statistically significantly higher mean ASES total scores at W24 and W52 post treatment than subjects in the corticosteroid group ( $<<0.05)$.

Discussion: Injection of fresh, uncultured, unmodified, autologous adipose derived regenerative cells is a safe and effective treatment of sPTRCT. Larger trials are needed to verify this result.
\end{abstract}

Trial registration: Clinicaltrials.gov ID NCT02918136. Registered September 28, 2016.

Level of evidence: Level I; prospective, randomized, controlled trial. 


\section{BACKGROUND}

Partial-thickness rotator cuff tears (PTRCT) are a common cause of shoulder paint, loss of function and occupational disability [1-3]. Cadaveric and magnetic resonance imaging (MRI) studies reported the incidence of PTRCT between 13\% and $25 \%$, with an increasing incidence with age [4-6]. The etiology and pathogenesis of PTRCT are multifactorial, and comprise several intrinsic factors (including age-related hypocellularity and decreased tissue vascularity) as well as extrinsic factors (including subacromial impingement, glenohumeral instability, internal impingement and trauma) [1-3].

According to the Guideline on Optimizing the Management of Rotator Cuff Problems of the American Academy of Orthopedic Surgeons [7, 8] the strength of recommendation for or against many nonoperative treatment options for rotator cuff tears and rotator cuff related symptoms (including activity modification, exercise programs, use of non-steroidal antiinflammatory drugs and corticosteroid injections) has remained "inconclusive". Subacromial injection of corticosteroid, provides short-term pain relief, but may not modify the course of the disease [9]. Steroid injections, commonly used in clinical practice, are not without risks. Dexamethasone (which has a 25 times higher antiinflammatory potency than hydrocortisone [10]) may induce non-tenocyte differentiation of human tendon stem cells, potentially leading to tendon rupture [11].

Most authors agree that surgical treatment of sPTRCT is generally indicated in patients with failure of nonoperative management for 3-6 months $[1,3]$. Surgical intervention, while generally successful, has some drawbacks, including the potential for complications, a lengthy recovery, and some authors report that it may not be better than conservative management [12].

Over the past decade, stem cell injection therapy has emerged as a promising treatment for a litany of conditions. In animal models, injections of adult stem cells isolated from adipose tissue into pathologic rotator cuff tissues has been shown to produce a positive biological response. The reported beneficial effects included decreased number of inflammatory cells, improved regeneration of tendons with less scarred healing, improved collagen fiber arrangement, higher load-tofailure and higher tensile strength of the treated tendons [1317]. However, corresponding clinical studies have not yet been reported.

Recent studies demonstrate the advantages of newer proprietary methods for harvesting and isolating stem cells [18-21]. The present study evaluated the safety and efficacy of treating sPTRCT which did not respond to traditional nonoperative care for six weeks with a single injection of UAADRCs isolated at point of care. The hypotheses were that (i) treatment of sPTRCT with UA-ADRCs does not result in any serious adverse event in the twelve months post treatment, and (ii) compared to subjects who received a single subacromial injection of corticosteroid, participants who received injection of UA-ADRCs show better function of the shoulder and greater reduction in pain at 24 and 52 weeks post treatment.

\section{METHODS}

\section{Study design}

This is a first in vivo, two center, prospective, open-label, randomized controlled trial comparing UA-ADRCs injection and corticosteroid injection for the management of symptomatic PTRCT not responsive to six weeks of traditional nonoperative care. All subjects were recruited from Sanford Orthopedics \& Sports Medicine Sioux Falls (Sioux Falls, SD, USA) and Sanford Orthopedics \& Sports Medicine Fargo (Fargo, ND, USA) between December 2016 and May 2017. Figure 1 shows the flow of subjects through this study according to the CONSORT statement [22], and Table 1 the schedule of enrollment, interventions and assessments according to SPIRIT [23]. Because this was a first-in-human trial, the study was designed as open-label trial, with treatment up to 30 days after screening and randomization. Subjects were allowed to withdraw their informed consent to participate in this study at any time.

\section{Ethics}

This study has received approval from the Institutional Review Board of Sanford Health (Sanford IRB \#3 registration number 00007985) in accordance with the Declaration of Helsinki, and Investigational Device Exemption from the U.S. Food and Drug Administration (FDA) (no. 16956). The trial was registered on September 28, 2016 at Clinicaltrials.gov, with ID NCT02918136.

\section{Participants}

Adults aged 30-75 years (both female and male) with clinical symptoms of rotator cuff tendinopathy including but not limited to pain, muscle weakness, or active-limited range of motion who had not responded to traditional nonoperative care for at least six weeks were eligible for inclusion. All subjects had MRI scans that demonstrated PTRCT > 50\% in the supraspinatus tendon (partial-thickness articular side tear, partial-thickness bursal-side tear or interstitial tear, respectively); MRI scans were reviewed by both the radiologists ( $\mathrm{MH}$ and $\mathrm{MH}$ ) and the treating physicians ([JLH and ML) participating in this study. The full list of inclusion and exclusion criteria is shown in Table 2 . The vast majority of subjects assessed for eligibility to be enrolled in this study were representative for citizens of U.S. Midwestern metropolitan areas. Subjects in both groups were recruited from the same population over the same period of time. 


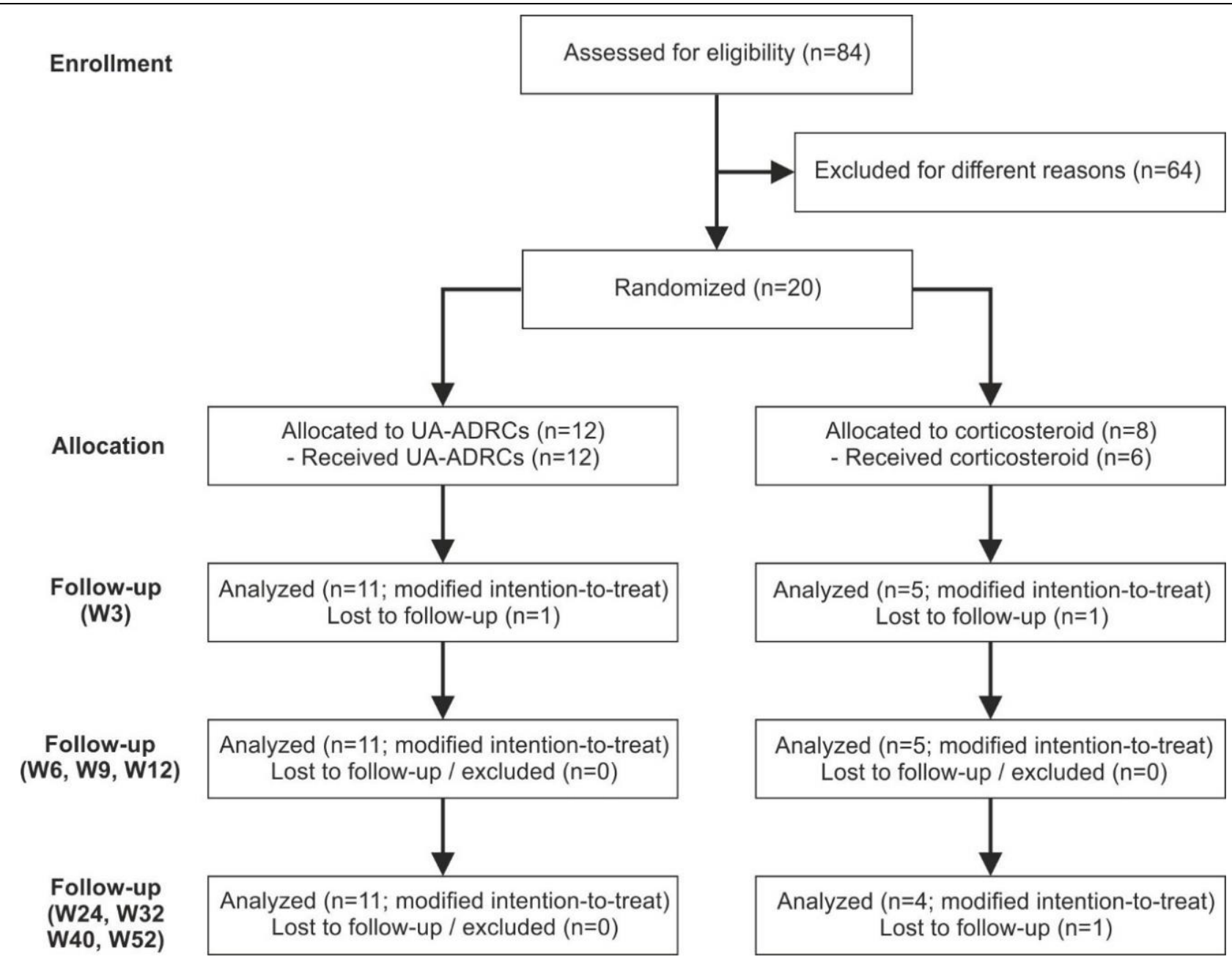

Figure 1 Flow of patients in this study according to CONSORT [22].

\section{Randomization and blinding}

A total of 84 subjects suffering from sPTRCT were assessed. Before randomization, 64 of 84 subjects assessed for eligibility chose to withdraw, declined to sign consent, or were excluded because they did not meet the inclusion or exclusion criteria. The remaining 20 subjects were randomly assigned to receive a single injection of UA-ADRCs $(n=12)$ or a single injection of methylprednisolone $(n=8)$. Randomization was performed using a computerized random-number generator to formulate subject allocation. The 20 subjects were randomized into six blocks. The person who determined whether a subject was eligible for inclusion in the study was unaware, when this decision was made, of which group the subject would be allocated to. One subject in the UA-ADRCs group was treated but excluded from the study immediately after treatment because the cell product failed to meet release criteria. Furthermore, two subjects in the corticosteroid group withdraw consent after randomization but prior to treatment, and another subject in this group withdraw consent shortly after treatment. Accordingly, the modified intention-to-treat (mITT) population comprised $n=11$ subjects in the UAADRCs group and $n=5$ subjects in the corticosteroid group (Figure 1).

One subject in the corticosteroid group developed a fullthickness rotator cuff tear after the examination that took place twelve weeks after treatment and was lost to follow-up. This resulted in full analysis of $11 / 11(100 \%)$ of the subjects in the UA-ADRCs group and $4 / 5(80 \%)$ of the subjects in the corticosteroid group (mITT population) (Figue 1). Missing data of the subject who was lost to follow-up were handled using the Last Observation Carried Forward method [24].

Characteristics of subjects in the mITT population at baseline are displayed in Table 3 .

The subjects, physicians who performed the treatment and assessors who performed baseline and follow-up examinations were not blinded in this study. On the other hand, the physicians who analyzed MRI scans were blinded 
Table 1 Schedule of enrollment, interventions and assessments during the present study according to SPIRIT [23]. Abbreviations: D, day; W, week; UA-ADRCs, fresh, uncultured, unmodified, autologous adipose derived regenerative cells; A, age; G, gender; BH, body height; BW, body weight; O, occupation; L, leisure activities. *, week after treatment. **, the baseline MRI scan of one subject was performed 36 days before screening.

\begin{tabular}{|c|c|c|c|c|c|c|c|}
\hline \multirow[b]{3}{*}{ Timepoint } & \multicolumn{6}{|c|}{ Study period } & \multirow{3}{*}{$\begin{array}{c}\text { Close-out } \\
\text { W52* }\end{array}$} \\
\hline & \multirow{2}{*}{$\begin{array}{c}\text { Enrollment / } \\
\text { Allocation } \\
\text { D0 }\end{array}$} & \multicolumn{2}{|c|}{ Post-allocation } & \multicolumn{3}{|c|}{ Follow-up } & \\
\hline & & D0-D2 & D8-D29 & $\begin{array}{l}\text { W3*, W6*, } \\
\text { W9*, W12* }\end{array}$ & W24* & $\mathrm{W} 32 *, \mathrm{~W} 40^{*}$ & \\
\hline \multicolumn{8}{|l|}{ Enrollment } \\
\hline Clinical evaluation & $\mathrm{X}$ & & & & & & \\
\hline Eligibility screen & $X$ & & & & & & \\
\hline Allocation & $\mathrm{X}$ & & & & & & \\
\hline MRI scan & & $\mathrm{X}^{* *}$ & & & $\mathrm{X}$ & & $\mathrm{X}$ \\
\hline \multicolumn{8}{|l|}{ Interventions } \\
\hline Injection of UA-ADRCs & & & $\mathrm{X}$ & & & & \\
\hline Injection of orticosteroid & & & $\mathrm{X}$ & & & & \\
\hline \multicolumn{8}{|l|}{ Assessments } \\
\hline $\mathrm{A}, \mathrm{G}, \mathrm{BH}, \mathrm{BW}, \mathrm{O}, \mathrm{L}$ & $\mathrm{X}$ & & & & & & \\
\hline Safety & & & $\mathrm{X}$ & $\mathrm{X}$ & $\mathrm{X}$ & $\mathrm{X}$ & $\mathrm{X}$ \\
\hline ASES & $X$ & & & $X$ & $\mathrm{X}$ & $X$ & $\mathrm{X}$ \\
\hline SF-36 & $\mathrm{X}$ & & & $\mathrm{X}$ & $\mathrm{X}$ & $\mathrm{X}$ & $X$ \\
\hline Pain & $X$ & & & $\mathrm{X}$ & $\mathrm{X}$ & $\mathrm{X}$ & $\mathrm{X}$ \\
\hline
\end{tabular}

\section{Interventions}

Subjects in the UA-ADRCs group had an outpatient syringe liposuction procedure performed by a licensed physician using a modified Coleman method [25]. To this end, either the periumbilical abdominal area, bilateral flanks or medial thigh, respectively, were surgically disinfected. Then, local anesthesia was achieved by infiltrating the subcutaneous adipose tissue with averaged $316 \pm 25 \mathrm{ml}$ (mean \pm standard error of the mean; SEM; range, 175-400) of modified Klein tumescent solution [26] (1000 mL lactated Ringer's solution (E8000; Braun Medical, Irvine, CA, USA), $50 \mathrm{ml}$ of $1 \%$ lidocaine (Hospira, Lake Forest, IL, USA) and $1 \mathrm{~cm}^{3}$ of 1:1000 epinephrine (Hospira)). Twenty minutes later, a stab incision was produced, and lipoaspiration was performed using a 4hole blunt tipped cannula $(3 \mathrm{~mm} \times 150 \mathrm{~mm})$ (Shippert Medical Technologies, Centennial, CO, USA) and a $60 \mathrm{~cm}^{3}$ Luer-Lock syringe (VAC160, Merit Medical, South Jordan, UT, USA). After liposuction, manual pressure was applied to the wounds. Then, the wounds were closed using adhesive bandage strips (Curity or Dermacea Abdominal Pad; Covedian, Mansfield, MA, USA).

The harvested lipoaspirate $(50 \mathrm{ml}$ per subject $)$ was processed with the Transpose RT / Matrase system (InGeneron, Houston, TX, USA) [18-21] to isolate UAADRCs. The lipoaspirate was divided into two aliquots of 25 $\mathrm{ml}$ each. Then, each aliquot was incubated together with Matrase Reagent (InGeneron) for 30 minutes. The latter was performed in the Transpose RT processing unit under agitation at $37^{\circ} \mathrm{C}$ according to the manufacturer's instructions. The total procedure time was 70 minutes. The average cell yield (i.e., the number of cells isolated per gram of tissue) was 2.3 $\pm 0.2 \times 10^{5} \mathrm{cells} / \mathrm{g}$, and the average cell viability was $88 \pm 3 \%$ (all data are related to the mITT population). Details of the final cell suspension are provided in Table 4.

No more than two hours after the lipoaspirate was harvested, each subject in the UA-ADRCs group received a single injection of averaged $11.4 \times 10^{6} \mathrm{UA}-\mathrm{ADRCs}$ in $5 \mathrm{~mL}$ liquid (mITT population). Subjects in the corticosteroid group received a single injection of $80 \mathrm{mg}$ of methylprednisolone (40 $\mathrm{mg} / \mathrm{ml} ; 2 \mathrm{ml}$ ) plus $3 \mathrm{ml}$ of $0.25 \%$ bupivacaine. Corticosteroid injections were made into the subacromial space and injections of UA-ADRCs were made into the tendon defect. All injections were made by a qualified physician under ultrasound guidance.

\section{Outcome measurements and assessments}

The primary clinical outcome was the occurrence of adverse events, defined as any untoward or unfavorable medical occurrence in a subject, including any abnormal sign, symptom or disease, temporally associated with the subject's participation in this study, whether or not considered related to the subject's participation in this study. Possible adverse events, the occurrence of which was specifically considered, were fever, bleeding, bruising, persistent swelling at the injection site, tenderness at the injection site, pain at the injection site, infection at the injection site, redness at the injection site, lightening of the skin around the injection site, joint infection, inflammatory flare, thinning of the skin and soft tissue around the injection site, tendon weakening, shoulder pain, worsening shoulder pain and nerve damage. Occurrence of adverse events was assessed immediately after treatment (i.e., injection of UA-ADRCs or corticosteroid) and three weeks (W3), W6, W9, W12, W24, W32, W40 and W52 post treatment 
Table 2 Inclusion and exclusion criteria of subjects with symptomatic, partial-thickness rotator cuff tear enrolled in the present study.

\begin{tabular}{l}
\hline Inclusion criteria \\
\hline Males and females $30-75$ years of age. \\
Clinical symptoms consistent with a rotator cuff lesion including but \\
not limited to pain, muscle weakness, or active-limited range of \\
motion (AROM).
\end{tabular}

Subjects who have not responded to physical therapy treatments for at least six weeks.

Subjects with $>70 \%$ passive range of motion (PROM).

Diagnosed with $>50 \%$ tear to supraspinatus muscle or $<5 \mathrm{~mm}$ separation assessed by MRI.

Diagnosed with a partial-thickness rotator cuff tear.

The ability of subjects to give appropriate consent.

\section{Exclusion criteria}

Age $<30$ or $>75$

Insufficient amount of subcutaneous tissue to allow recovery of 50 $\mathrm{mL}$ of lipoaspirate.

History of systemic malignant neoplasms within last 5 years.

History of local neoplasm within the last 6 months and any history of local neoplasm at site of administration.

Subject is receiving immunosuppressant therapy or has known immunosuppressive or severe autoimmune disease that requires chronic immunosuppressive therapy (e.g., human immunodeficiency virus, systemic lupus erythematosus, etc.).

Subjects who are known to be HIV positive.

Patients who have received a corticosteroid injection in rotator cuff site within last 3 months.

Severe arthrosis of the glenohumeral or acromioclavicular joint.

Irreparable rotator cuff tear (including rotator cuff tear arthropathy).

Fatty atrophy above Grade 2 in affected shoulder.

Previous shoulder surgeries in affected shoulder.

Any contraindication to MRI scan according to MRI guidelines, or unwillingness to undergo MRI procedures.

History of tobacco use within the last 3 months.

Patient is on an active regimen of chemotherapy.

Patients with a documented history of liver disease or an ALT value $>400$.

Allergy to sodium citrate of any "caine" type of local anesthetic.

Patient is pregnant or breast feeding.

Subject is, in the opinion of the investigator or designee, unable to comply with the requirements of the study protocol or is unsuitable for the study for any reason. This includes completion of Patient Reported Outcome instruments.

Subject is currently participating in another clinical trial that has not yet completed its primary endpoint.

Subject is part of a vulnerable population who, in the judgment of the investigator, is unable to give Informed Consent for reasons of incapacity, immaturity, adverse personal circumstances or lack of autonomy. This may include: individuals with mental disability, persons in nursing homes, children, impoverished persons, persons in emergency situations, homeless persons, nomads, refugees, and those incapable of giving informed consent. Vulnerable populations also may include members of a group with a hierarchical structure such as university students, subordinate hospital and laboratory personnel, employees of the sponsor, members of the armed forces, and persons kept in detention.

Uncooperative patients or those with neurological/psychiatric disorders who are incapable of following directions or who are predictably unwilling to return for follow-up examinations.
Secondary clinical outcomes were changes in the (i) American Shoulder and Elbow Surgeons (ASES) Society standardized shoulder assessment form (ASES total score), (ii) RAND Short Form-36 total score (c.f. [27, 28]), (iii) Visual Analogue Scale for pain (VAS pain score) and (iv) size of the PTRCT measured on MRI scans as a function of time compared to baseline.

The ASES assessment form shows strong correlation with multiple rotator cuff specific scores, and has excellent reliability, construct validity and responsiveness $[29,30]$. The RAND Short Form-36 is a global measure of health-related quality of life that measures eight scales: physical functioning, role physical, bodily pain, general health, vitality, social functioning, role emotional and mental health [31].

Pain was assessed in two ways. First, the ASES pain score was scaled from 10 (pain as bad as it can be) to 0 (no pain at all). The specific question asked was about the "Intensity of Pain" in the shoulder with no time reference given to guide the subject. Accordingly, the question suggested the overall severity of pain when experienced. Second, a validated VAS was used. The VAS is a very specific term that classically refers to an unidimensional line (i.e. an unmarked line that is $100 \mathrm{~mm}$ in length) to measure intensity of pain. The ends are defined as the extreme limits of the parameter to be measured orientated from the left (worst) to the right (best). This scale was embedded in the RAND Short Form-36 in the present study. The specific question asked was "What is your level of pain in your shoulder today?".

MRI evaluation of PTRCT was performed in the coronal, sagittal and axial plane with $\mathrm{T} 2$ proton density weighted, fat saturated (PD FS) sequences (repetition time (TR) between 2375 and $3917 \mathrm{~ms}$; echo time (TE) between 34 and $72 \mathrm{~ms}$; NEX between 1.0 and 2.0; slice thickness between 3.0 and 4.0 $\mathrm{mm}$, image size $320 \times 320$ or $512 \times 512$, respectively). MRI scans were performed using a MAGNETOM Skyra 3T (Siemens Medical Solutions USA, Malvern, PA, USA) or Signa Architect 3.0 T (GE Healthcare, Waukesha, WI, USA), respectively. The size of the PTRCT was measured in all three directions, and the tear volume was calculated as an ellipsoid from these data [32].

The ASES total score, RAND Short Form-36 total score and VAS pain score were assessed at baseline (BL) and W3, W6, W9, W12, W24, W32, W40 and W52 post treatment; MRI scans were performed at BL, W24 and W52 post treatment.

\section{Power analysis}

Because this was a first-in-human trial with occurrence of adverse events as primary clinical outcome, a small sample size was selected that was not based on a power analysis. Rather, the ultimate goal of this study was to collect safety data that are sufficient to develop an appropriate pivotal study after six months of data collection for all subjects. This pivotal study, which finally will include 246 subjects with sPTRCT, is now recruiting (Clinicaltrials.gov ID NCT03752827 [33]). 
Table 3 Characteristics of included subjects at baseline (modified intention-to-treat population). *, body height was not taken from one subject in the corticosteroid group.

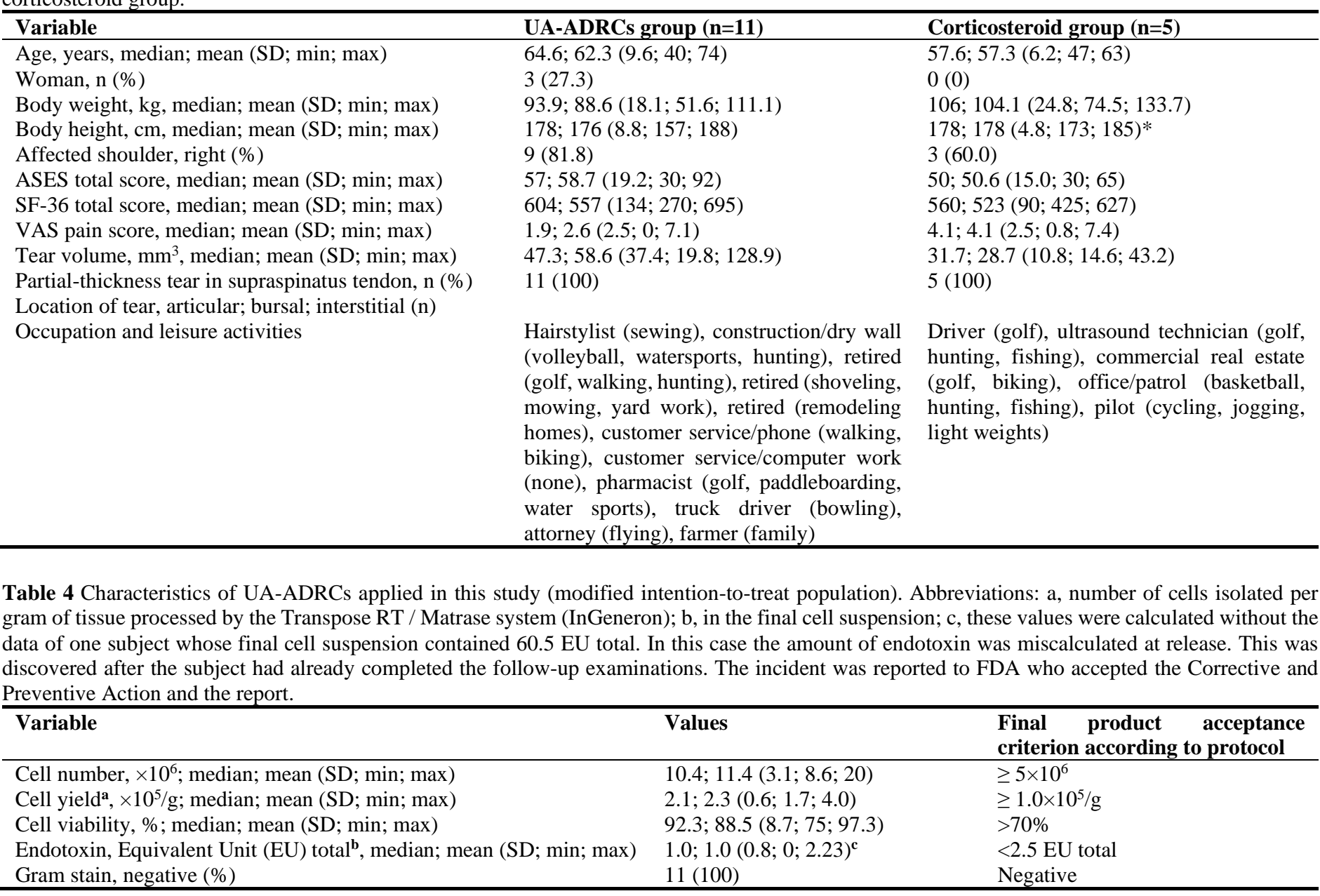

\section{Statistical analysis}

Mean and standard error of the mean (SEM) of all investigated variables (ASES total score, RAND Short Form-36 total score, VAS pain score and size of PTRCT) were calculated. Differences between the groups were tested using two-way repeated measures ANOVA, with subject-specific values obtained at BL, W24 and W52 post treatment as matched data (values obtained at $\mathrm{W} 3, \mathrm{~W} 6, \mathrm{~W} 9, \mathrm{~W} 12, \mathrm{~W} 32$ and $\mathrm{W} 40$ post treatment were not considered in the statistical analysis). Post hoc Bonferroni tests were used for pairwise comparisons. In all analyses, an effect was considered statistically significant if its associated $\mathrm{p}$ value was smaller than 0.05 . Calculations were performed using GraphPad Prism (Version 8.0.0 for Windows; GraphPad Software, San Diego, CA, USA).

\section{RESULTS}

\section{Safety of treating symptomatic, partial-thickness rotator cuff tear with UA-ADRCs}

The results are summarized in Table 5. The total number of
TEAEs was 34 in the UA-ADRCs group and 12 in the corticosteroid group. All subjects in both groups reported experiencing at least one TEAE. The number of subjects with $1 / 2 / 3 / 4 / 5 / 6 / 7$ TEAEs was $1 / 3 / 4 / 2 / 0 / 0 / 1$ in the UA-ADRCs group (3.1 \pm 0.5 ; median, 3$)$ and $2 / 1 / 1 / 0 / 1 /$ $0 / 0$ in the corticosteroid group $(2.4 \pm 0.8$; median, 2$)$. These data were not significantly different between the groups (Mann-Whitney test; $\mathrm{p}=0.405$ ).

Of note, the majority of TEAEs were reported in one subject. Four different subjects in the UA-ADRCs group (one subject each on Day 22 [D22], D44, D168 and D224 post treatment) and four different subjects in the corticosteroid group (one subject each at D43, D49, D64 and D158 post treatment) reported musculoskeletal pain. Furthermore, in the UA-ADRCs group two different subjects reported pain in extremity (one subject at D208 post treatment, and another subject at D3 and again at D120 post treatment, with different extremities affected at D3 and D120), another two different subjects reported abdominal pain (both immediately post treatment), and another two different subjects reported dyspnoea (one subject each on D27 and D364 post treatment).

The number of TEAEs classified as mild / moderate / severe 
was $21 / 10 / 3$ in the UA-ADRCs group and $8 / 4 / 0$ in the corticosteroid group. The three severe TEAEs in the UAADRCs group were myocardial infarction (one subject on D91 and again at D126 post treatment) and musculoskeletal pain (another subject at D224 post treatment). None of the severe TEAEs were related to treatment.

The relationship of TEAEs to treatment classified as Not related / Unlikely / Possible / Probable / Definite was 26 / 4 / $4 / 0$ / 0 in the UA-ADRCs group and $7 / 3$ / 2/0/0 in the corticosteroid group. Those TEAEs that were classified as possible in the UA-ADRCs group were mild abdominal pain (two subjects immediately post treatment; most probably because of the liposuction procedure), moderate pain in extremity (another subject on D3 post treatment) and mild viral upper respiratory tract infection (another subject on D14 post treatment), and in the corticosteroid group moderate musculoskeletal pain (two subjects on D43 and D49 post treatment).

Except for one subject, the amount of endotoxin in the final cell suspension of all subjects in the UA-ADRCs group was below 2.5 Equivalent Units (EU) total (Table 4), which was the final product acceptance criterion according to protocol. The final cell suspension of one subject contained a total of 60.5 EU of endotoxin. In this subject, the amount of endotoxin was miscalculated at release: this was discovered after the subject had already completed the follow-up examinations. The incident was reported to FDA who accepted the Corrective and Preventive Action and the report. The following TEAEs were reported for this subject: musculoskeletal pain on Day 22 (D22) post treatment (mild; relationship to treatment classified as unlikely) and myocardial infarction on D91 and D126 post treatment (severe; not related).

Table 5. Number of treatment emergent adverse events (TEAE) and number of subjects with TEAE by system organ class and preferred term (coded with the Medical Dictionary for Regulatory Activities (MedDRA) Version 19.1 [34]). Abbreviations: A, adverse event category; D, days post treatment; G, Group; S, severity; P, probability: C, corticosteroid group; U, UA-ADRCs group.; VURTI, viral upper respiratory tract infection; COPD, chronic obstructive pulmonary disease.

\begin{tabular}{|c|c|c|c|c|c|c|c|c|c|}
\hline $\mathbf{A}$ & $\mathbf{D}$ & $\mathbf{G}$ & $\mathbf{S}$ & $\mathbf{P}$ & $\mathbf{A}$ & D & $\mathbf{G}$ & $\mathbf{S}$ & $\mathbf{P}$ \\
\hline \multicolumn{5}{|l|}{ Cardiac disorders } & \multicolumn{5}{|c|}{ Musculoskeletal and connective tissue disorders } \\
\hline Coron. artery disease & 21 & $\mathrm{C}$ & Mild & Not related & Arthralgia (knee) & 8 & $\mathrm{U}$ & Mild & Not related \\
\hline Myocardial infarction & 91 & $\mathrm{U}$ & Severe & Not related & Arthralgia (hip) & 114 & $\mathrm{C}$ & Mild & Not related \\
\hline Myocardial infarction & 126 & $\mathrm{U}$ & Severe & Not related & Back pain & 33 & $\mathrm{U}$ & Mild & Not related \\
\hline \multicolumn{5}{|c|}{ Gastrointestinal disorders } & Musculoskeletal pain & 22 & $\mathrm{U}$ & Mild & Unlikely \\
\hline Abdominal discomfort & 86 & $\mathrm{U}$ & Mild & Not related & Musculoskeletal pain & 43 & $\mathrm{C}$ & Moderate & Possible \\
\hline Abdominal pain & 0 & $\mathrm{U}$ & Mild & Possible & Musculoskeletal pain & 44 & $\mathrm{U}$ & Mild & Unlikely \\
\hline Abdominal pain & 0 & $\mathrm{U}$ & Mild & Possible & Musculoskeletal pain & 49 & $\mathrm{C}$ & Moderate & Possible \\
\hline Dental necrosis & 35 & $\mathrm{U}$ & Moderate & Unlikely & Musculoskeletal pain & 64 & $\mathrm{C}$ & Mild & Unlikely \\
\hline Dysphagia & 242 & $\mathrm{U}$ & Moderate & Not related & Musculoskeletal pain & 158 & $\mathrm{C}$ & Mild & Unlikely \\
\hline Abdominal discomfort & 86 & $\mathrm{U}$ & Mild & Not related & Musculoskeletal pain & 168 & $\mathrm{U}$ & Mild & Not related \\
\hline \multicolumn{5}{|c|}{ General disorders and administration site conditions } & Musculoskeletal pain & 224 & $\mathrm{U}$ & Severe & Not related \\
\hline Chest pain & 149 & $\mathrm{C}$ & Mild & Not related & Pain in extremity & 3 & $\mathrm{U}$ & Moderate & Possible \\
\hline \multicolumn{5}{|l|}{ Infections and infestations } & Pain in extremity & 120 & $\mathrm{U}$ & Mild & Not related \\
\hline Bronchitis & 231 & $\mathrm{U}$ & Moderate & Not related & Pain in extremity & 208 & $\mathrm{U}$ & Mild & Not related \\
\hline Diverticulitis & 299 & $\mathrm{U}$ & Moderate & Not related & Tendonitis & 58 & $\mathrm{U}$ & Moderate & Not related \\
\hline Pharyngitis & 4 & $\mathrm{C}$ & Mild & Unlikely & \multicolumn{5}{|c|}{ Neoplasms benign, malignant and unspecified (incl cysts and polyps } \\
\hline Pharyngitis & 133 & $\mathrm{U}$ & Mild & Not related & Seborrhoeic keratosis & 126 & $\mathrm{U}$ & Mild & Not related \\
\hline Sinusitis & 49 & $\mathrm{U}$ & Mild & Not related & \multicolumn{5}{|l|}{ Psychiatric disorders } \\
\hline Staphylococcal infect. & 141 & $\mathrm{U}$ & Mild & Not related & Alcoholism & 139 & $\mathrm{U}$ & Mild & Not related \\
\hline VURTI & 14 & $\mathrm{U}$ & Mild & Possible & \multicolumn{5}{|c|}{ Renal and urinary disorders } \\
\hline \multicolumn{5}{|c|}{ Injury, poisoning and procedural complications } & Dysuria & 19 & $\mathrm{U}$ & Mild & Not related \\
\hline Concussion & 40 & $\mathrm{U}$ & Moderate & Not related & \multicolumn{5}{|c|}{ Respiratory, thoracic and mediastinal disorders } \\
\hline Contusion & 29 & $\mathrm{U}$ & Mild & Not related & COPD & 296 & $\mathrm{U}$ & Moderate & Not related \\
\hline Ligament sprain & 35 & $\mathrm{C}$ & Mild & Not related & Cough & 147 & $\mathrm{C}$ & Mild & Not related \\
\hline Tendon rupture & 30 & $\mathrm{C}$ & Moderate & Not related & Dyspnoea & 27 & $\mathrm{U}$ & Moderate & Unlikely \\
\hline Tooth fracture & 164 & $\mathrm{C}$ & Moderate & Not related & Dyspnoea & 364 & $\mathrm{U}$ & Moderate & Not related \\
\hline \multicolumn{5}{|c|}{ Skin and subcutaneous tissue disorders } & Rhinitis allergic & 65 & $\mathrm{U}$ & Mild & Not related \\
\hline Nail discolouration & 126 & $\mathrm{U}$ & Mild & Not related & & & & & \\
\hline \multicolumn{5}{|l|}{ Vascular disorders } & & & & & \\
\hline Essential hypertension & 129 & $\mathrm{U}$ & Mild & Not related & & & & & \\
\hline
\end{tabular}

Gram stain of the final cell suspension of all subjects in the UA-ADRCs group showed a negative result prior to cell administration. Furthermore, 14-day cultures of 10 out of the 11 final cell suspensions of these subjects displayed a negative finding. The 14-day culture of the final cell suspension of one subject evidenced the presence of Propionibacterium acnes. The TEAEs reported for this subject were viral upper respiratory tract infection on D14 post treatment (mild; 
possible), dyspnoea on D27 post treatment (moderate; unlikely), rhinitis allergic on D65 post treatment (mild; not related) and essential hypertension on D129 post treatment (mild; not related).

Collectively, these data demonstrate that treatment of sPTRCT with UA-ADRCs isolated from lipoaspirate at point of care is safe. The risks connected with treatment of sPTRCT with UA-ADRCs were not greater than those connected with treatment of sPTRCT with corticosteroid injection.

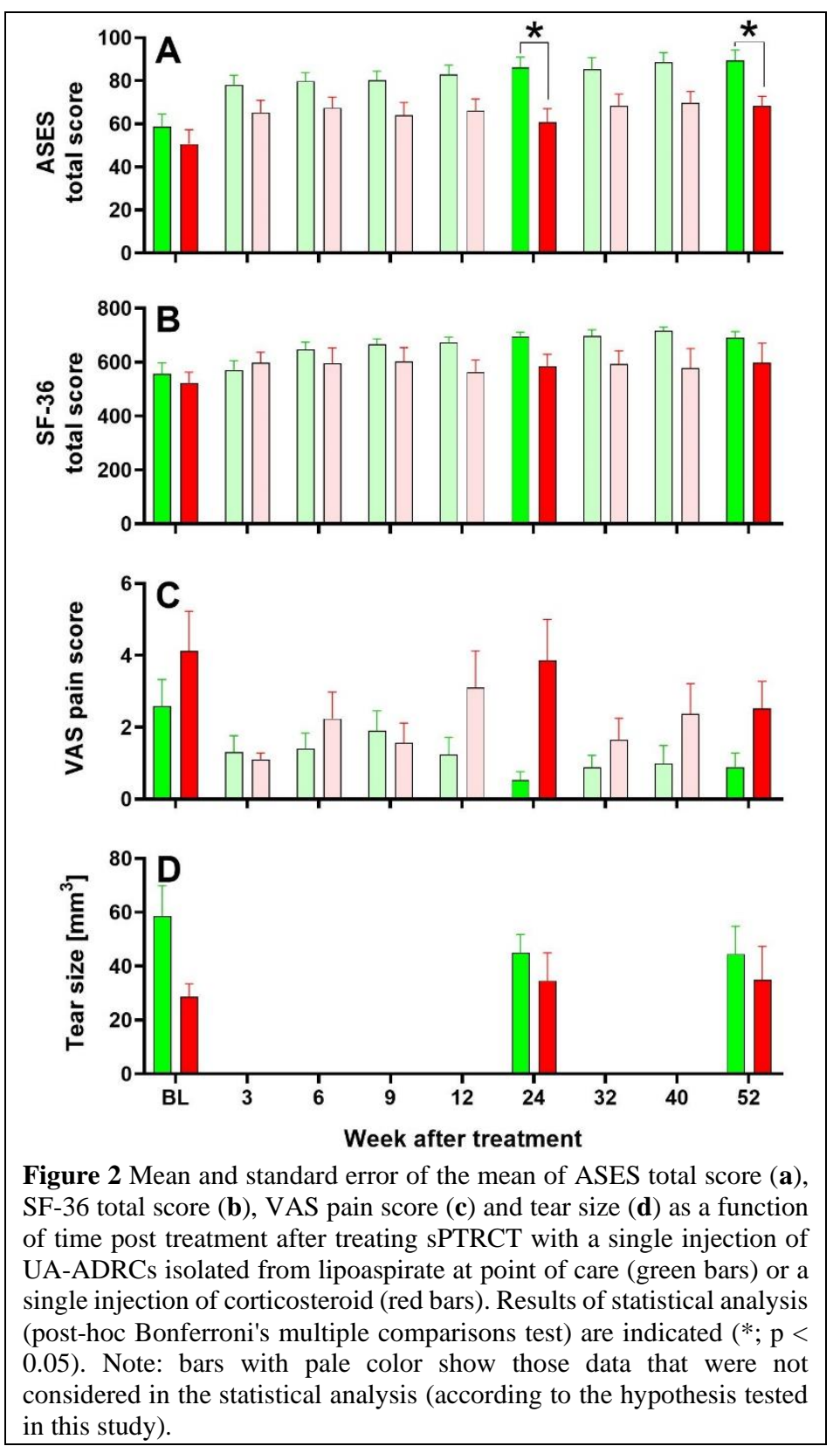

Efficacy of treating symptomatic, partial-thickness rotator cuff tear with $U A-A D R C s$

With regard to efficacy of treating SPTRCT with respectively a single injection of UA-ADRCs isolated from adipose tissue at point of care or a single injection of corticosteroid (secondary clinical endpoint of this study), Figure 2 shows mean and SEM of ASES total score, RAND Short Form-36 total score, VAS pain score and size of the PTCRT as a function of time post treatment. Results of the statistical analysis ( $\mathrm{p}$ values) of these data are summarized in Table 6 .

Table 6. Results of the statistical analysis of the efficacy data. P values $<0.05$ are given boldface.

\begin{tabular}{|c|c|c|c|c|}
\hline $\begin{array}{l}\text { Analysis } \\
\text { Variable }\end{array}$ & $\begin{array}{ll} & \begin{array}{l}\text { ASES } \\
\text { total } \\
\text { score }\end{array} \\
\end{array}$ & $\begin{array}{l}\text { SF-36 } \\
\text { total } \\
\text { score } \\
\end{array}$ & $\begin{array}{l}\text { VAS } \\
\text { pain } \\
\text { score }\end{array}$ & $\begin{array}{l}\text { Tear } \\
\text { size }\end{array}$ \\
\hline \multicolumn{5}{|c|}{ Two-way RM ANOVA } \\
\hline $\mathrm{P}_{\text {Time }}$ & $<0.001$ & 0.006 & 0.040 & 0.811 \\
\hline $\mathrm{P}_{\text {Treatment }}$ & 0.017 & 0.088 & 0.011 & 0.237 \\
\hline $\mathrm{P}_{\text {Time }} \times$ Treatment & 0.199 & 0.425 & 0.249 & 0.305 \\
\hline PSubject & 0.008 & 0.005 & $\mathbf{0 . 0 2 4}$ & $<0.001$ \\
\hline \multicolumn{5}{|c|}{ Post-hoc Bonferroni's multiple comparisons test } \\
\hline $\mathrm{P}_{\mathrm{BL}}$ & $>0.009$ & $>0.009$ & 0.847 & 0.090 \\
\hline $\mathrm{P}_{\mathrm{W} 24}$ & $\mathbf{0 . 0 3 3}$ & 0.195 & 0.124 & $>0.999$ \\
\hline $\mathrm{P}_{\mathrm{W} 52}$ & 0.022 & 0.835 & 0.306 & $>0.999$ \\
\hline
\end{tabular}

The mean ASES total score of the subjects in the UAADRCs group increased from 58.7 \pm 5.8 (mean \pm SEM) at BL to $86.1 \pm 4.9$ at $\mathrm{W} 24$ and $89.4 \pm 4.9$ at W52 post treatment, and of the subjects in the corticosteroid group from $50.6 \pm 6.7$ at BL to $60.8 \pm 6.2$ at $\mathrm{W} 24$ and $68.4 \pm 4.4$ at W52 post treatment (Fig. 2A). Differences between the groups at W24 and W52 were statistically significant $(\mathrm{p}<0.05)$.

The mean RAND Short Form-36 total score of the subjects in the UA-ADRCs group increased from $557 \pm 40.1$ at BL to $696 \pm 15.7$ at W24 and $691 \pm 22.6$ at W52 post treatment, and of the subjects in the corticosteroid group from $523 \pm 40.4$ at BL to $586 \pm 44.0$ at W24 and $599 \pm 72.2$ at W52 post treatment (Fig. 2B). Differences between the groups were not statistically significant $(\mathrm{p}>0.05)$.

The mean VAS pain score of the subjects in the UA-ADRCs group decreased from $2.6 \pm 0.7$ at BL to $0.5 \pm 0.2$ at W24 and $0.9 \pm 0.4$ at W52 post treatment, and of the subjects in the corticosteroid group from $4.1 \pm 1.1$ at BL to $3.9 \pm 1.1$ at W24 and $2.5 \pm 0.8$ at W52 post treatment (Fig. 2C). As in case of the mean RAND Short Form-36, the total score differences between the groups were not statistically significant $(\mathrm{p}>$ $0.05)$.

The mean tear size of the subjects in the UA-ADRCs group decreased from $58.6 \pm 11.3 \mathrm{~mm}^{3}$ at BL to $45.0 \pm 6.8 \mathrm{~mm}^{3}$ at W24 and $44.5 \pm 10.3 \mathrm{~mm}^{3}$ at W52 post treatment, and of the subjects in the corticosteroid group increased from $28.7 \pm 4.8$ $\mathrm{mm}^{3}$ at BL to $34.6 \pm 10.4$ at W24 and $35.0 \pm 12.4$ at W52 post treatment (Fig. 2D). Again, the differences between the groups were not statistically significant $(p>0.05)$. 


\section{DISCUSSION}

To our knowledge, this is the first report of sPTRCT treated with UA-ADRCs. We evidenced the following key findings: (i) no severe adverse events related to the injection of UAADRCs in the twelve months after treatment; (ii) no greater risks than those connected with treatment of SPTRCT with corticosteroid injection; and (iii) subjects in the UA-ADRCs group showed statistically significantly higher mean ASES total scores at W24 and W52 post treatment than subjects in the corticosteroid group $(\mathrm{p}<0.05)$.

\section{Safety of treating symptomatic, partial-thickness rotator cuff tear with UA-ADRCs}

The excellent safety profile of treating SPTRCT with UAADRCs presented here, which was the primary clinical outcome of this study, was based on the following three pillars: (i) application of UA-ADRCs rather than other types of stem cells; (ii) enzymatic rather than non-enzymatic isolation of UA-ADRCs; and (iii) use of the Transpose RT / Matrase system (InGeneron; [18-21]) rather than other systems for enzymatic isolation of UA-ADRCs. With respect to the second and third pillar, Aronowitz and colleagues [35] proposed to judge a system or method for isolating UAADRCs by the following factors: nucleated cell count, nucleated cells per milliliter of tissue processed, cellular viability, level of residual enzymatic activity, data from flow cytometry and CFU-F assay, infection control, ease of use, cost to operate, and processing time. All of these aspects were addressed for the Transpose RT / Matrase system in detail in a recent experimental study [19].

\section{Efficacy of treating symptomatic, partial-thickness rotator cuff tear with UA-ADRCs}

Demonstration of the efficacy of treating sPTRCT with UAADRCs was only the secondary clinical outcome of this study, which justified the small sample of subjects and the limited selection of clinical examination methods. However, it is possible to draw a number of important conclusions from the available data.

The first conclusion addresses the impact of asking different questions about subjects' pain on the outcome of pain assessment (ASES pain score: "Intensity of Pain" in the shoulder with no time reference given to guide the subject; VAS: "What is your level of pain in your shoulder today?"). In this context, Figure 3 shows comparisons of the individual time course of ASES pain score and VAS pain score of select subjects in this study. Specifically, Figure 3A-C represents one subject each treated with UA-ADRCs and respectively substantial deviation between the two pain scores at baseline but fast recovery after treatment (Fig. 3A), almost identical pain scores and slower recovery after treatment (Fig. 3B), or dissociation between the two pain scores but no recovery over time (Fig. 3C). Figure 3D-F represents one subject each treated with corticosteroid and similar time course. In this sample of six (3 vs. 3) subjects, the key difference between the subjects treated with UA-ADRCs and those treated with corticosteroid was that the former showed significantly lower VAS pain scores at M24 than the latter $(0.9 \pm 0.4$ [UAADRCs] vs. $5.1 \pm 1.1$ [corticosteroid]; $\mathrm{p}=0.024$ [unpaired T test]), despite the fact that their mean VAS score was higher at baseline $(6.3 \pm 1.0$ [UA-ADRCs] vs. $3.4 \pm 1.4$ [corticosteroid]). These data do not only justify to present separate VAS pain scores (next to ASES pain scores as part of ASES total scores) in this study, but emphasize the need to precisely describe the questions that were asked when assessing subjects' pain in studies with pain as relevant endpoint.

The second conclusion that can be drawn from the results of the MRI analysis performed in this study (Fig. 2D) may suggest that treatment of sPTRCT with UA-ADRCs did not result in complete healing up to one year post treatment. However, a more differentiated consideration appears necessary in this regard. First of all, the question of complete healing would have required to analyze biopsies of the treated tendons, which was not covered by the study protocol. Second, the question must be asked whether complete healing can be expected in case of treating SPTRCT with $>50 \%$ tear with a single injection of UA-ADRCs. Particularly in case of tears with frayed margins, this may simply not be possible (evaluation of the margins of the tears would have required arthroscopic inspection, which was not covered by the study protocol). Third, in patients with PTRCT there is poor association between subject symptoms and degree of structural integrity of the rotator cuff [36, 37]. Accordingly, clinical improvement appears much more relevant than disappearance of PTRCT on MRI scans in treatment of SPTRCT, and the latter was achieved in this study using UAADRCs. In this regard Figure 4 shows comparisons of the individual time course of tear size and ASES total score of select subjects in this study; the corresponding MRI scans (coronal plane) are summarized in Figure 5. Specifically, Figure 4A-C represents one subject each treated with UAADRCs and excellent clinical outcome whose PTRCT showed respectively complete healing (Fig. 4A), partial healing (Fig. 4B), or initial healing followed by worsening over time (Fig. 4C). Figure 4D-F represents one subject each treated with corticosteroid whose PTRCT showed respectively almost complete healing (Fig. 4D) or worsening over time (Fig. 4E), or only a very small tear (Fig. 4F). In this sample of six (3 vs. 3) subjects, the key difference between the subjects treated with UA-ADRCs and those treated with corticosteroid was that the former showed better clinical improvement than the latter, despite the fact that their mean ASES score was slightly lower at baseline $(45 \pm 8.7$ [UA-ADRCs] vs. $53 \pm 6.5$ [corticosteroid]) and their mean tear size was significantly larger at baseline $\left(62 \pm 7.7 \mathrm{~mm}^{3}\right.$ [UA-ADRCs] vs. $23 \pm 5.0$ $\mathrm{mm}^{3}$ [corticosteroid]; $\mathrm{p}=0.012$ [unpaired $\mathrm{T}$ test]). 

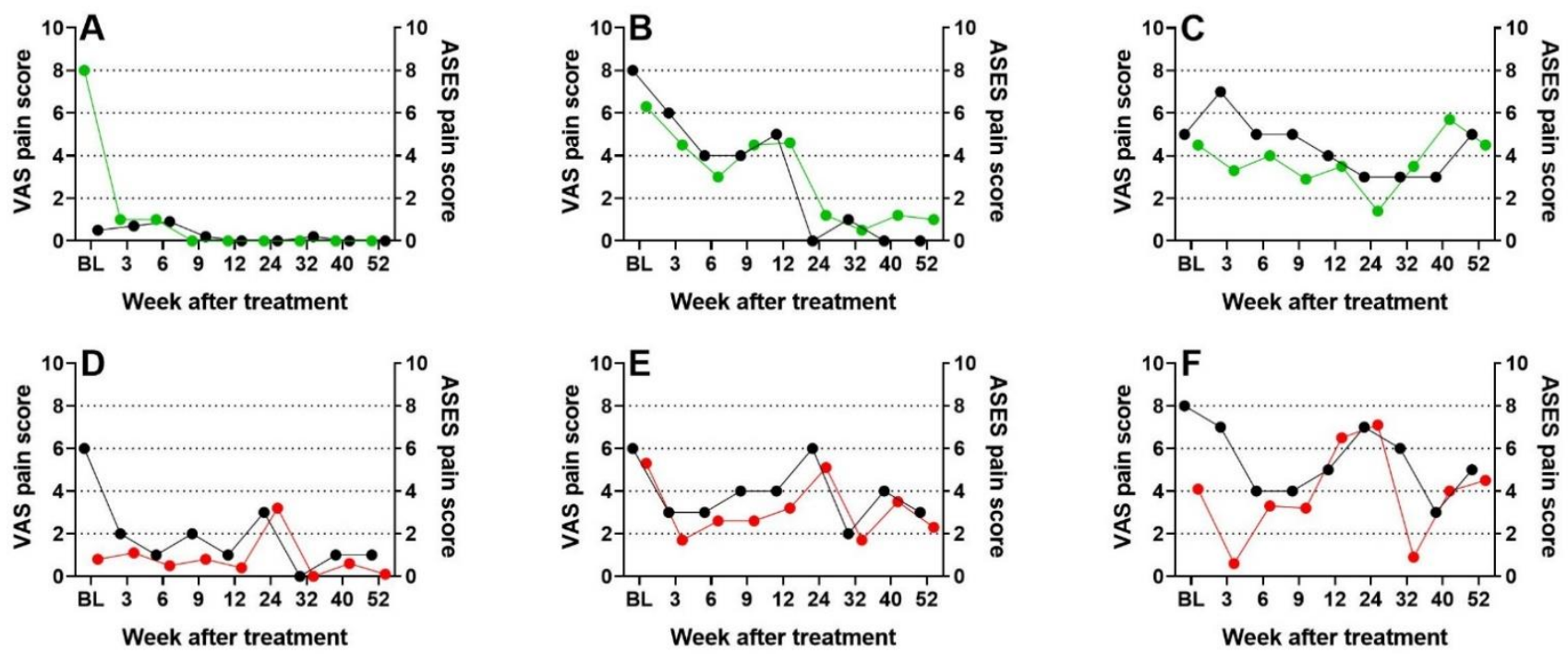

Figure 3 Individual ASES pain scores (black dots and right Y axis) and VAS pain scores (green and red dots and left Y axis) of select subjects treated with UA-ADRCs (A-C) or corticosteroid (D-F) in this study. Details are provided in the main text.
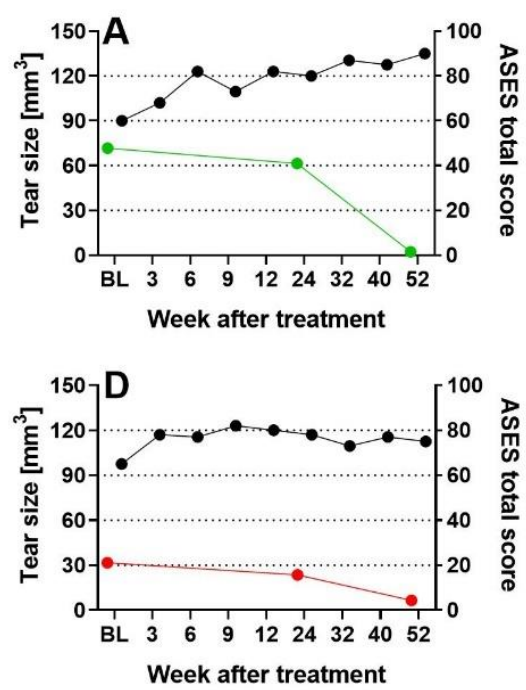
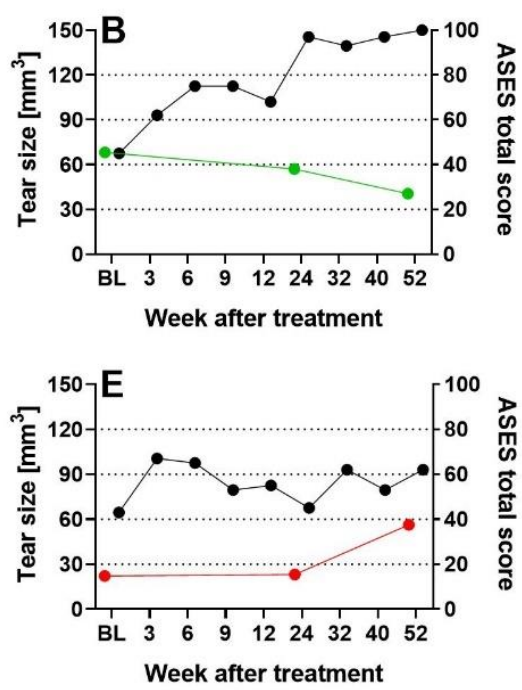
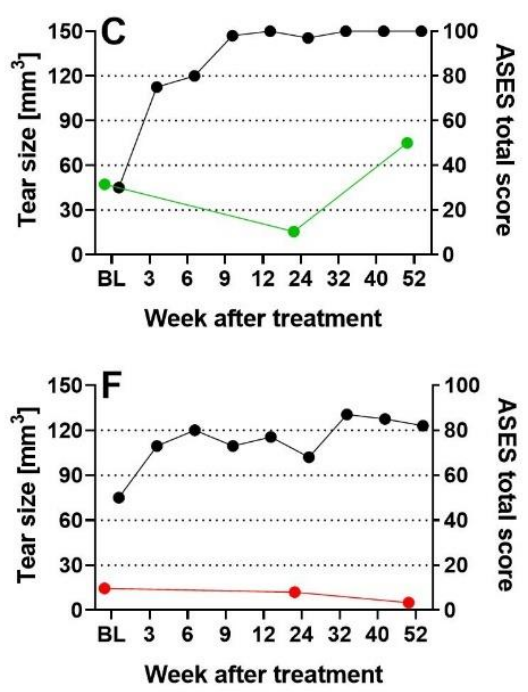

Figure 4 Individual ASES total scores (black dots and right $\mathrm{Y}$ axis) and tear size (green and red dots and left $\mathrm{Y}$ axis) of select subjects treated with UA-ADRCs (A-C) or corticosteroid (D-F) in this study. Details are provided in the main text.

Collectively, these findings point to mechanisms of action of UA-ADRCs in the treatment of sPTRCT beyond merely regenerating tendon tissue. However, it is crucial to bear in mind that, in contrast to ASCs, UA-ADRCs in principle cannot be labeled. Accordingly, it is not possible to experimentally (or even clinically) determine whether the following benefits of ASCs also apply to UA-ADRCs, although it is reasonable to hypothesize that this is indeed the case. Specifically, ASCs can stay locally, survive and engraft in the new host tissue into which the cells were applied [38], differentiate under guidance of the new microenvironment into cells of all three germ layers [19], integrate into and communicate within the new host tissue by forming direct cell-cell contacts [18], exchange genetic and epigenetic information through release of exosomes [18], participate in building new vascular structures in the host tissue [19, 21], positively influence the new host tissue by release of cytokines (among them vascular endothelial growth factor and insulinlike growth factor 1) [39], protect cells at risk in the new host tissue from undergoing apoptosis [39] and induce immune- 
modulatory and anti-inflammatory properties [40, 41]. In any case, the combination of these mechanisms of action apparently render UA-ADRCs more powerful in the treatment of sPTRCTs than corticosteroid.

Treatment of subjects suffering from sPTRCT with stem cells has been reported in several studies [42-46]. However, for a variety of reasons (no control group, no randomization, augmentation of rotator cuff repair with stem cells, arthroscopic evaluation of the rotator cuff before injection of stem cells, use of bone-marrow derived mesenchymal stem cells (BM-MSCs), use of ASCs rather than UA-ADRCs) most of these studies and their outcome cannot be compared with the present study (summarized in Table 7). In particular, in four of these studies [42-45] stem cells were used for augmenting athroscopic rotator cuff repair rather than serving as the only therapy (aside from physiotherapy and physical therapy after initial treatment). An exception is a study by Jo et al. [46], in which subjects suffering from sPTRCT were treated with injection of $1 \times 10^{7}$ ASCs (Group 1; n=3), $5 \times 10^{7}$ ASCs (Group 2;n=3) or $1 \times 10^{8}$ ASCs (Groups $3(n=3)$ and 4 $(\mathrm{n}=10))$, respectively. Liposuction was performed three weeks before injection. During a follow-up period of six months after treatment subjects in all groups showed a reduction in the mean Shoulder Pain and Disability Index (Group 1: from 43 to 18; Group 2: from 64 to 12; Group 3: from 75 to 16) and VAS pain score (Group 1: from 78 to 36; Group 2: from 90 to
28; Group 3: from 90 to 26) (all data are approximate values taken from graphical representation of data in [46]). Furthermore, subjects in Groups 2 and 3 showed an improvement in the Constant score during the follow-up period (Groups 2: from 62 to 76; Group 3: from 56 to 66). Statistical analysis did not included between groups comparison. Of note, all subjects in Groups 1 and 2 suffered from bursal-sided PTRCT whereas subjects in Group 3 suffered from articular-sided PTRCT, and no group showed complete healing as evaluated with MRI scans and arthoscopic inspection at the end of the follow-up period. In summary, the results by Jo et al. [46] are in line with the results of this study. However, they do not establish any advantage of ASCs over UA-ADRCs in the treatment of sPTRCT. Rather, one has to consider all the potential disadvantages of ASCs outlined above, which may also explain that the best results were obtained by Jo et al. [46] when injecting a number of ASCs that was a magnitude higher than the number of UA-ADRCs that was applied in this study. Furthermore, a recent publication by the Lancet Commission on Stem Cells and Regenerative Medicine [47] highlighted that, when performing long term expansion in culture, even under optimal conditions, cells are exposed to different kinds of stress (mechanic, oxidative) that could affect their safety as medicinal product (quoted from [47]).

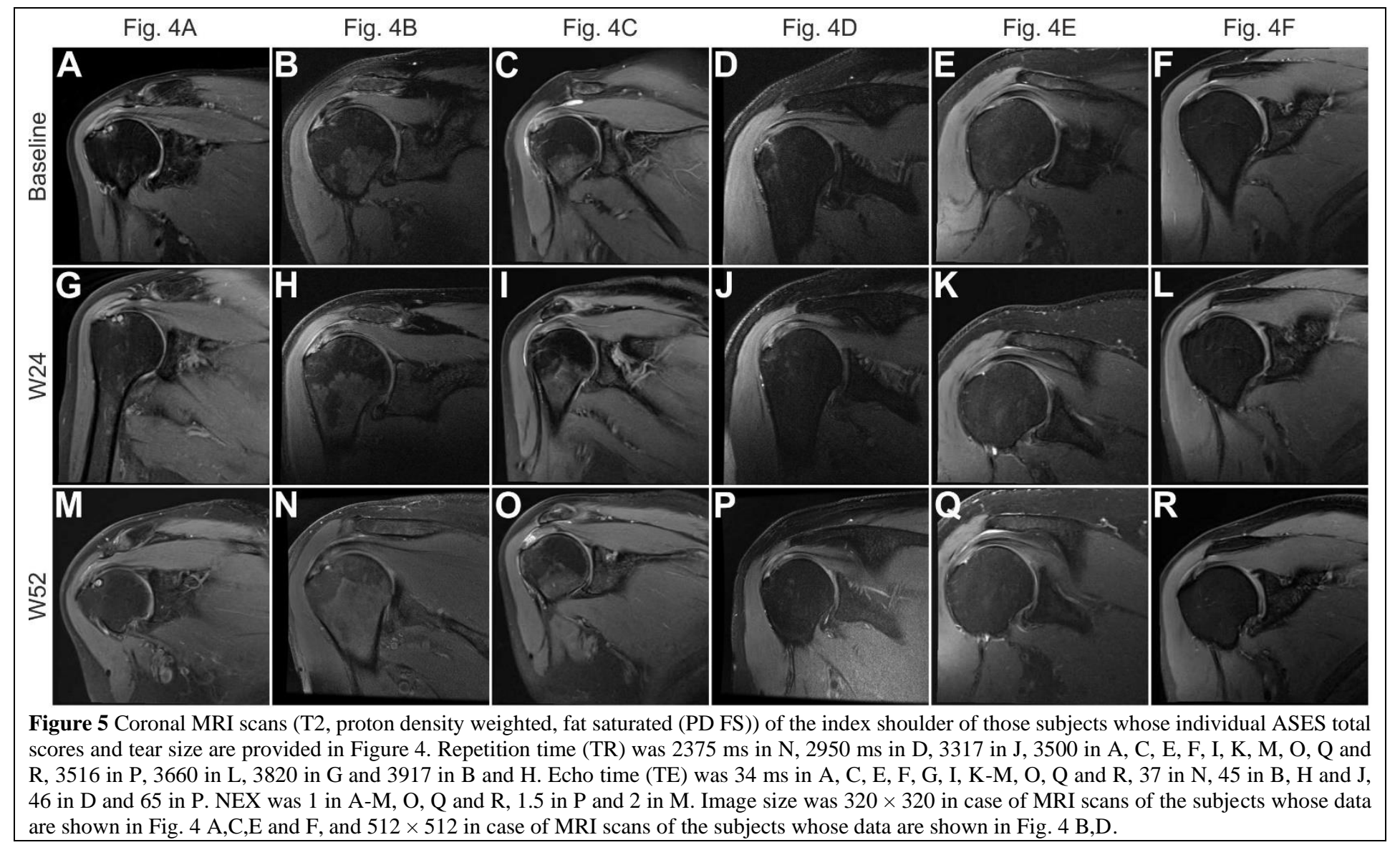


Table 7. Comparison of key characteristics of the present study with other studies on treatment of subjects suffering from sPTRCT with stem cells published in the literature. Abbreviations: RCT, randomized controlled trial; UA-ADRCs, fresh, uncultured, unmodified, autologous adipose derived regenerative cells; ASCs, adipose-derived stem cells; BM-MSCs, bone marrow-derived mesenchymal stem cells. * Different groups of subjects with different doses of ASCs, but no other control group.

\begin{tabular}{|c|c|c|c|c|c|c|}
\hline Characteristic & $\begin{array}{c}\text { This } \\
\text { study }\end{array}$ & {$[42]$} & [43] & {$[44]$} & {$[45]$} & {$[46]$} \\
\hline Use of stem cells as the sole therapy & Yes & No & No & No & No & Yes \\
\hline Control group & Yes & No & No & No & Yes & No* \\
\hline RCT & Yes & No & No & No & No & No \\
\hline Augmentation of rotator cuff repair with stem cells & No & Yes & Yes & Yes & Yes & No \\
\hline Arthroscopic evaluation of the rotator cuff before injection of stem cells & No & $\mathrm{n} / \mathrm{a}$ & $\mathrm{n} / \mathrm{a}$ & $\mathrm{n} / \mathrm{a}$ & $\mathrm{n} / \mathrm{a}$ & Yes \\
\hline Use of UA-ADRCs (1), ASCs (2) or BM-MSCs (3) & 1 & 3 & 3 & 3 & 1 & 2 \\
\hline
\end{tabular}

Some authors proposed bone marrow stimulation by drilling of holes into the proximal humerus at the footprint of arthroscopic rotator cuff repair [48, 49]. However, the real benefit of these procedures in which mesenchymal stem cells (MSCs) may serve to augment arthroscopic rotator cuff repair rather than the only therapy has yet to be demonstrated (c.f. [17]). The latter also applies to isolation of MSCs from the subacromial bursa, rotator cuff tendon, biceps tendon or synovial fluid, respectively, as proposed by some authors (reviewed in [17]). To our knowledge, application of such MSCs in the management of PTRCT have not yet been reported. In any case, it appears not possible to isolate MSCs from the subacromial bursa, rotator cuff tendon, biceps tendon and synovial fluid at point of care.

Many studies tested treatment of rotator cuff injuries and SPTRCT with platelet rich plasma (PRP) (see [50-57] for the most recent systematic reviews, meta-analyses and editorial comments in this regard). Whereas earlier systematic reviews and meta-analyses showed no (or almost no) benefit of augmenting arthroscopic rotator cuff repair with PRP [50-53], two very recent meta-analyses demonstrated that the use of PRP in rotator cuff repair resulted in improved functional outcomes, pain levels and healing rates compared to control $[54,55]$. In contrast, another very recent meta-analysis concluded that PRP injections may not be beneficial in nonoperative treatment of rotator cuff disease [57]. This is supported by a very recent, well-designed, double-blinded, randomized controlled trial on 80 adults with symptomatic isolated interstitial tears of the supraspinatus tendon which reported no improvement in clinical scores and tendon healing between injection of PRP or saline within the interstitial supraspinatus tears [58]. Even worse, injection of PRP was associated with more adverse events than injection of saline in the latter study [58]. One reason for the lack of benefit of PRP in nonoperative treatment of rotator cuff tears may be hypocellularity as an important intrinsic factor of the etiology and pathogenesis of PTRCT [1-3]. Considering the potential mismatch between growth factors released by PRP [59] and an insufficient number of stem cells in PTRCT [1-3] to be stimulated by these growth factors, both from a conceptual point of view and based on the data discussed here application of UA-ADRCs appears to be the better option for treating SPTRCT.

\section{Limitations}

Because this prospective, randomized, controlled trial on UAADRCs vs. corticosteroid for treating SPTRCT was a first-inhuman study with safety as primary clinical endpoint, it was designed as open-label pilot trial: thus, it has a number of inherent limitations. Specifically, only a small sample of subjects suffering from sPTRCT was investigated, only a limited number of clinical examination methods was applied, no power analysis was carried out, and neither the subjects nor the physicians who performed treatment and the assessors who performed baseline and follow-up examinations were blinded (only the physicians who analyzed the MRI scans were blinded). However, it was not the ultimate aim of this study to conclusively establish UA-ADRCs as treatment of sPTRCT. Rather, the ultimate aim of this study was to collect safety data that are sufficient to develop an appropriate pivotal study which finally will include 246 subjects with sPTRCT. This pivotal study, which is not affected by the limitations outlined above, is now recruiting (Clinicaltrials.gov ID NCT03752827 [37]) on the basis of the very encouraging pilot data presented in the present report.

\section{Conclusions}

The results of this study suggest that the use of UA-ADRCs in subjects with sPTRCT is safe and effective, leading to a significant improvement in shoulder function, without adverse effects. Clinicians should consider UA-ADRCs instead of injection of corticosteroids or PRP, and prior to surgical intervention, in the management of sPTRCT.

\section{Acknowledgements}

We express our gratitude to all the subjects who consented to participate in this study. We also thank everyone at Sanford Health (Fargo, ND, USA and Sioux Falls, SD, USA) and Sanford Research for their contribution to the study operations.

\section{Funding}

This study was supported by InGeneron, Inc. (Houston, TX, USA). The sponsor of the study did not have any influence on data collection, analysis or publication. No constraints were placed on publication of the data.

Availability of data and materials

The datasets used and analyzed during the current study are available 
from the corresponding author on reasonable request, taking into account any confidentiality.

\section{Authors' contributions}

JLH, TRF, JW, MH, MH, GEW, EUA and LM conceived the study and participated in the design of the study. JLH and ML performed the treatments. $\mathrm{MH}$ and $\mathrm{MH}$ analyzed the MRI scans. CS performed the statistical analysis and drafted the manuscript. JPF, NM and CA helped to draft the manuscript. All authors read and approved the final manuscript.

\section{Ethics approval and consent to participate}

Ethical approval for this study was granted by the Institutional Review Board of Sanford Health (Sanford IRB \#3 registration number 00007985), and all subjects gave informed consent.

\section{References}

1. Matava MJ, Purcell DB, Rudzki JR. Partial-thickness rotator cuff tears. Am J Sports Med. 2005;33(9):1405-17. doi: 10.1177/0363546505280213.

2. Via AG, De Cupis M, Spoliti M, Oliva F. Clinical and biological aspects of rotator cuff tears. Muscles Ligaments Tendons J. 2013;3(2):70-9. doi: 10.11138/mltj/2013.3.2.070.

3. Matthewson G, Beach CJ, Nelson AA, Woodmass JM, Ono Y, Boorman RS, Lo IK, Thornton GM. Partial thickness rotator cuff tears: current concepts. Adv Orthop. 2015;2015:458786. doi: $10.1155 / 2015 / 458786$.

4. Cotton RE, Rideout DF. Tears of the humeral rotator cuff. J Bone Joint Surg 1964;46B(2):314-28.

5. Fukuda H. Partial-thickness rotator cuff tears: a modern view on Codman's classic. J Shoulder Elbow Surg. 2000;9(2):163-8.

6. Sher JS, Uribe JW, Posada A, Murphy BJ, Zlatkin MB. Abnormal findings on magnetic resonance images of asymptomatic shoulders. J Bone Joint Surg Am. 1995;77(1):10-5. doi: 10.2106/00004623199501000-00002.

7. https://www.aaos.org/research/guidelines/rcp_summary.pdf [cited 09 December 2019].

8. Checketts JX, Scott J, Gordon J, Jones J, Horn J, Farabough M, Whitener J, Boose M, Vassar M. An evaluation of the rotator cuff repair research pipeline. Orthop J Sports Med. 2018;6(11):2325967118805731. doi: 10.1177/2325967118805731.

9. Coombes BK, Bisset L, Vicenzino B. Efficacy and safety of corticosteroid injections and other injections for management of tendinopathy: a systematic review of randomised controlled trials. Lancet. 2010;376(9754):1751-67. doi: 10.1016/S01406736(10)61160-9.

10. https://clincalc.com/Corticosteroids/ [cited 9 December 2019].

11. Zhang J, Keenan C, Wang JH. The effects of dexamethasone on human patellar tendon stem cells: implications for dexamethasone treatment of tendon injury. J Orthop Res. 2013;31(1):105-10. doi: 10.1002/jor.22193.

12. Kukkonen J, Joukainen A, Lehtinen J, Mattila KT, Tuominen EK, Kauko T, Aärimaa V. Treatment of non-traumatic rotator cuff tears: A randomised controlled trial with one-year clinical results. Bone Joint J. 2014;96-B(1):75-81. doi: 10.1302/0301-620X.96B1.32168.

13. Kim SH, Chung SW, Oh JH. Expression of insulin-like growth factor type 1 receptor and myosin heavy chain in rabbit's rotator cuff muscle after injection of adipose-derived stem cell. Knee Surg Sports Traumatol Arthrosc. 2014;22(11):2867-73. doi: 10.1007/s00167-013-2560-6.

14. Valencia Mora M, Antuña Antuña S, García Arranz M, Carrascal MT, Barco R. Application of adipose tissue-derived stem cells in a rat rotator cuff repair model. Injury. 2014;45 Suppl 4:S22-7. doi:

\section{Consent for publication}

We have obtained consent for publication.

\section{Competing interests}

JLH, TRF, JW, MH, MH and ML are employees of Sanford Health, a minority shareholder of InGeneron, Inc. (Houston, TX, USA), which is the manufacturer and distributor of the Transpose RT / Matrase system that was investigated in this study. GEW is employed as Chief Scientific Officer at InGeneron, Inc.. CS has served as consultant to SciCoTec (Gruenwald, Germany), the majority shareholder of InGeneron, Inc.. CA is managing director of InGeneron GmbH (Munich, Germany) which is owned by InGeneron, Inc.. EA is Executive Chair of InGeneron, Inc., and Chairman of the Board of SciCoTec.

10.1016/S0020-1383(14)70006-3.

15. Chen HS, Su YT, Chan TM, Su YJ, Syu WS, Harn HJ, Lin SZ, Chiu SC. Human adipose-derived stem cells accelerate the restoration of tensile strength of tendon and alleviate the progression of rotator cuff injury in a rat model. Cell Transplant. 2015;24(3):509-20. doi: $10.3727 / 096368915 \times 686968$.

16. Gumucio JP, Flood MD, Roche SM, Sugg KB, Momoh AO, Kosnik PE, Bedi A, Mendias CL. Stromal vascular stem cell treatment decreases muscle fibrosis following chronic rotator cuff tear. Int Orthop. 2016;40(4):759-64. doi: 10.1007/s00264-015-2937-x.

17. Tsekes D, Konstantopoulos G, Khan WS, Rossouw D, Elvey M, Singh J. Use of stem cells and growth factors in rotator cuff tendon repair. Eur J Orthop Surg Traumatol. 2019;29(4):747-57. doi: 10.1007/s00590-019-02366-x.

18. Alt EU, Schmitz C, Bai X. Fundamentals of Stem cells: why and how patients' own adult stem cells are the next generation of medicine. Preprints. 2019:2019040200. doi: 10.20944/preprints201904.0200.v1.

19. Winnier GE, Valenzuela N, Peters-Hall J, Kellner J, Alt C, Alt EU. Isolation of adipose tissue derived regenerative cells from human subcutaneous tissue with or without the use of an enzymatic reagent. PLoS One. 2019;14(9):e0221457. doi: 10.1371/journal.pone.0221457.

20. Solakoglu Ö, Götz W, Kiessling MC, Alt C, Schmitz C, Alt EU. Improved guided bone regeneration by combined application of unmodified, fresh autologous adipose derived regenerative cells and plasma rich in growth factors: A first-in-human case report and literature review. World J Stem Cells. 2019;11(2):124-46. doi: 10.4252/wjsc.v11.i2.124.

21. Haenel A, Ghosn M, Karimi T, Vykoukal J, Shah D, Valderrabano M, Schulz DG, Raizner A, Schmitz C, Alt EU. Unmodified autologous stem cells at point of care for chronic myocardial infarction. World J Stem Cells. 2019;11(10):831-58. doi: 10.4252/wjsc.v11.i10.831.

22. Boutron I, Altman DG, Moher D, Schulz KF, Ravaud P; CONSORT NPT Group. CONSORT Statement for Randomized Trials of Nonpharmacologic Treatments: A 2017 Update and a CONSORT Extension for Nonpharmacologic Trial Abstracts. Ann Intern Med. 2017;167(1):40-47. doi: 10.7326/M17-0046.

23. Chan AW, Tetzlaff JM, Altman DG, Laupacis A, Gøtzsche PC, Krleža-Jerić K, Hróbjartsson A, Mann H, Dickersin K, Berlin JA, Doré CJ, Parulekar WR, Summerskill WS, Groves T, Schulz KF, Sox HC, Rockhold FW, Rennie D, Moher D. SPIRIT 2013 statement: defining standard protocol items for clinical trials. Ann Intern Med. 2013;158(3):200-7. doi: 10.7326/0003-4819-158-3201302050-00583.

24. European Medicine Agency. Guideline on missing data in 
confirmatory clinical trials, 2010 [cited 09 December 2019]. Available

from:

www.ema.europa.eu/docs/en_GB/document_library/Scientific_gui deline/2010/09/WC500096793.pdf.

25. Coleman WP 4th, Hendry SL 2nd. Principles of liposuction. Semin Cutan Med Surg. 2006;25:138-44. doi: 10.1016/j.sder.2006.06.006.

26. Klein JA. Tumescent technique for local anesthesia improves safety in large-volume liposuction. Plast Reconstr Surg.1993;92:1085-98.

27. Ware J, Donald Sherbourne C. The MOS 36-item short-form health survey (SF-36). I. Conceptual framework and item selection. Med Care 1992;30(6):473-83.

28. Hays RD, Sherbourne CD, Mazel RM. The RAND 36-Item Health Survey 1.0. Health Econ 1993;2(3):217-27.

29. Angst F, Schwyzer HK, Aeschlimann A, Simmen BR, Goldhahn J. Measures of adult shoulder function: Disabilities of the Arm, Shoulder, and Hand Questionnaire (DASH) and its short version (QuickDASH), Shoulder Pain and Disability Index (SPADI), American Shoulder and Elbow Surgeons (ASES) Society standardized shoulder assessment form, Constant (Murley) Score (CS), Simple Shoulder Test (SST), Oxford Shoulder Score (OSS), Shoulder Disability Questionnaire (SDQ), and Western Ontario Shoulder Instability Index (WOSI). Arthritis Care Res. 2011;63 Suppl 11:S174-88. doi: 10.1002/acr.20630.

30. Wylie JD, Beckmann JT, Granger E, Tashjian RZ. Functional outcomes assessment in shoulder surgery. World J Orthop. 2014;5(5):623-33. doi: 10.5312/wjo.v5.i5.623.

31. Lins L, Carvalho FM. SF-36 total score as a single measure of healthrelated quality of life: Scoping review. SAGE Open Med. 2016;4:2050312116671725.

32. Bronstein IN, Semendjajew KA. Handbook of Mathematics (3rd ed). Harri Deutsch, Frankfurt/Main, 1985.

33. Hurd J. Autologous adult adipose-derived regenerative cell injection into chronic partial-thickness rotator cuff tears. ClinicalTrials.gov Identifier: NCT03752827 [cited 09 December 2019]. Available from: https://www.clinicaltrials.gov/ct2/show/NCT03752827.

34. https://www.meddra.org/ [cited 09 December 2019].

35. Aronowitz JA, Lockhart RA, Hakakian CS, Birnbaum ZE. Adipose stromal vascular fraction isolation: A head-to-head comparison of 4 cell separation systems \#2. Ann Plast Surg. 2016;77(3):354-62. doi: 10.1097/SAP.0000000000000831.

36. Brockmeyer M, Schmitt C, Haupert A, Kohn D, Lorbach O. Limited diagnostic accuracy of magnetic resonance imaging and clinical tests for detecting partial-thickness tears of the rotator cuff. Arch Orthop Trauma Surg. 2017;137(12):1719-24. doi: 10.1007/s00402-0172799-3.

37. Edwards A, Chepeha J, Jones A, Sheps DM, Beaupré L. Can clinical assessment differentiate partial thickness rotator cuff tears from full thickness rotator cuff tears? A secondary analysis. Disabil Rehabil. 2019; Feb 8: Epub ahead of print. doi: 10.1080/09638288.2018.1563637.

38. Bai X, Yan Y, Coleman M, Wu G, Rabinovich B, Seidensticker M, Alt E. Tracking long-term survival of intramyocardially delivered human adipose tissue-derived stem cells using bioluminescence imaging. Mol Imaging Biol. 2011;13(4):633-45. doi: 10.1007/s11307-010-0392-Z

39. Sadat S, Gehmert S, Song YH, Yen Y, Bai X, Gaiser S, Klein H, Alt E. The cardioprotective effect of mesenchymal stem cells is mediated by IGF-I and VEGF. Biochem Biophys Res Commun. 2007;363(3):674-9. doi: 10.1016/j.bbrc.2007.09.058.

40. González MA, Gonzalez-Rey E, Rico L, Büscher D, Delgado M. Adipose-derived mesenchymal stem cells alleviate experimental colitis by inhibiting inflammatory and autoimmune responses. Gastroenterology. 2009;136(3):978-89. doi: 10.1053/j.gastro.2008.11.041

41. Leto Barone AA, Khalifian S, Lee WP, Brandacher G.
Immunomodulatory effects of adipose-derived stem cells: fact or fiction? Biomed Res Int. 2013;2013:383685. doi: $10.1155 / 2013 / 383685$.

42. Ellera Gomes JL, da Silva RC, Silla LMR, Abreu MR, Pellanda R. Conventional rotator cuff repair complemented by the aid of mononuclear autologous stem cells. Knee Surg Sports Traumatol Arthrosc. 2012;20(2):373-7. doi: 10.1007/s00167-011-1607-9.

43. Hernigou P, Flouzat Lachaniette CH, Delambre J, Zilber S, Duffiet $\mathrm{P}$, Chevallier N, Rouard H. Biologic augmentation of rotator cuff repair with mesenchymal stem cells during arthroscopy improves healing and prevents further tears: a case-controlled study. Int Orthop. 2014;38(9):1811-8. doi: 10.1007/s00264-014-2391-1.

44. Havlas V, Kotaška J, Koníček P, Trč T, Konrádová Š, Kočí Z, Syková E. Pouziti kultivovanych lidskych autolognich kmenovych bunek kostni drene pri rekonstrukci ruptury rotatorove manzety studie bezpecnosti metody, predbezne vysledky [Use of cultured human autologous bone marrow stem cells in repair of a rotator cuff tear: preliminary results of a safety study] [Article in Czech]. Acta Chir Orthop Traumatol Cech. 2015;82(3):229-34.

45. Kim YS, Sung CH, Chung SH, Kwak SJ, Koh YG. Does an injection of adipose-derived mesenchymal stem cells loaded in fibrin glue influence rotator cuff repair outcomes? a clinical and magnetic resonance imaging study. Am J Sports Med. 2017 Jul;45(9):2010-8. doi: $10.1177 / 0363546517702863$.

46. Jo CH, Chai JW, Jeong EC, Oh S, Kim PS, Yoon JY, Yoon KS. Intratendinous injection of autologous adipose tissue-derived mesenchymal stem cells for the treatment of rotator cuff disease: a first-in-human trial. Stem Cells. 2018;36(9):1441-50. doi: 10.1002/stem.2855

47. Cossu G, Birchall M, Brown T, De Coppi P, Culme-Seymour E, Gibbon S, Hitchcock J, Mason C, Montgomery J, Morris S, Muntoni F, Napier D, Owji N, Prasad A, Round J, Saprai P, Stilgoe J, Thrasher A, Wilson J. Lancet Commission: Stem cells and regenerative medicine. Lancet. 2018;391(10123):883-910. doi: 10.1016/S0140-6736(17)31366-1.

48. Jo CH, Shin JS, Park IW, Kim H, Lee SY. Multiple channeling improves the structural integrity of rotator cuff repair. Am J Sports Med. 2013;41(11):2650-7. doi: 10.1177/0363546513499138.

49. Taniguchi N, Suenaga N, Oizumi N, Miyoshi N, Yamaguchi H, Inoue $\mathrm{K}$, Chosa $\mathrm{E}$. Bone marrow stimulation at the footprint of arthroscopic surface-holding repair advances cuff repair integrity. J Shoulder Elbow Surg. 2015;24(6):860-6. doi: 10.1016/j.jse.2014.09.031

50. Cai YZ, Zhang C, Lin XJ. Efficacy of platelet-rich plasma in arthroscopic repair of full-thickness rotator cuff tears: a metaanalysis. J Shoulder Elbow Surg. 2015;24(12):1852-9. doi: 10.1016/j.jse.2015.07.035.

51. Warth RJ, Dornan GJ, James EW, Horan MP, Millett PJ. Clinical and structural outcomes after arthroscopic repair of full-thickness rotator cuff tears with and without platelet-rich product supplementation: a meta-analysis and meta-regression. Arthroscopy. 2015;31(2):306-20. doi: 10.1016/j.arthro.2014.09.007.

52. Fu CJ, Sun JB, Bi ZG, Wang XM, Yang CL. Evaluation of plateletrich plasma and fibrin matrix to assist in healing and repair of rotator cuff injuries: a systematic review and meta-analysis. Clin Rehabil. 2017;31(2):158-72. doi: 10.1177/0269215516634815.

53. Miranda I, Sánchez-Alepuz E, Lucas FJ, Carratalá V, GonzálezJofre CA. Use of platelet-rich plasma in the treatment of rotator cuff pathology. What has been scientifically proven? Rev Esp Cir Ortop Traumatol. 2017;61(4):249-58. doi: 10.1016/j.recot.2017.03.001.

54. Hurley ET, Lim Fat D, Moran CJ, Mullett H. The efficacy of plateletrich plasma and platelet-rich fibrin in arthroscopic rotator cuff repair: a meta-analysis of randomized controlled trials. Am J Sports Med. 2019;47(3):753-61. doi: 10.1177/0363546517751397.

55. Jazrawi LM, Baron SL. Editorial commentary: sprinkle some pixie 
dust on it-are we really any better at understanding the benefits of platelet-rich plasma for rotator cuff pathology? Arthroscopy. 2019;35(5):1592-3. doi: 10.1016/j.arthro.2019.02.009.

56. Han C, Na Y, Zhu Y, Kong L, Eerdun T, Yang X, Ren Y. Is plateletrich plasma an ideal biomaterial for arthroscopic rotator cuff repair? A systematic review and meta-analysis of randomized controlled trials. J Orthop Surg Res. 2019;14(1):183. doi: 10.1186/s13018-0191207-9.

57. Hurley ET, Hannon CP, Pauzenberger L, Fat DL, Moran CJ, Mullett $\mathrm{H}$. Nonoperative treatment of rotator cuff disease with platelet-rich plasma: a systematic review of randomized controlled trials. Arthroscopy. 10.1016/j.arthro.2018.10.115

58. Schwitzguebel AJ, Kolo FC, Tirefort J, Kourhani A, Nowak A, Gremeaux V, Saffarini M, Lädermann A. Efficacy of platelet-rich plasma for the treatment of interstitial supraspinatus tears: a doubleblinded, randomized controlled trial. Am J Sports Med. 2019;47(8):1885-92. doi: 10.1177/0363546519851097.

59. Murray IR, LaPrade RF, Musahl V, Geeslin AG, Zlotnicki JP, Mann BJ, Petrigliano FA. Biologic treatments for sports injuries II Think tank-current concepts, future research, and barriers to advancement, Part 2: rotator cuff. Orthop J Sports Med. 201631;4(3):2325967116636586. doi: 10.1177/2325967116636586. 